\title{
Pain and Depression in a Cohort of Underserved, Community-Dwelling Primary Care Patients
}

\author{
Janine E. Janosky, PhD, Jeannette E. South-Paul, MD, and Chyongchiou J. Lin, PhD
}

Purpose: Almost 17\% of the US population exhibits a major depressive disorder in their lifetimes. Prevalence data show that whites experience depression earlier than African Americans, and women have a higher prevalence than men. Less is known regarding depression among underserved minority populations. The goal of our study was to examine the relationship of depression and associated self-reported conditions in participants enrolled in a community-based research registry, a substantial number of whom were underrepresented minorities.

Metbods: This study used a research registry of community members who had expressed interest in participating in health education projects conducted by the Center for Primary Care Community-Based Research. The patients received care at 10 family health centers. Participants were surveyed regarding family history of depression/anxiety and associated symptoms. Descriptive analyses, univariate analyses, and logistic regressions were used.

Results: The population ( $\mathrm{N}=2421$ ) included women (72.2\%), African Americans (54.9\%), and reported good or very good general health (68.9\%). Comorbid pain was found, with headache as the predominant complaint. Compared with nonwhites, whites had a significantly higher prevalence of current depression $(26.3 \%$ vs. $23.8 \% ; P=.01)$, current anxiety $(25.5 \%$ vs. $16.6 \%)$, and current headache (14.2\% vs. $11.2 \%)$. Whites also had a higher prevalence of a family history of depression $(38.4 \% \mathrm{vs}$. $32.1 \%)$ and anxiety (8.9\% vs. $7.7 \%)$ and of taking depression $(22.4 \%$ vs. $14.8 \%)$ and anxiety (15.8\% vs. $7.8 \%)$ medications. However, nonwhites had a higher prevalence of leg pain (18.8\% vs. $14.9 \%)$ but a lower prevalence of headache (11.2\% vs. $14.2 \%)$.

Conclusions: Pain was common in patients with comorbid behavioral conditions. Headache was more common in whites, whereas leg pain was more common in nonwhites. Physicians should screen for depression and anxiety in patients with headache and other pain symptoms. (J Am Board Fam Med 2012;25:300-307.)

Keywords: Community Medicine, Depression, Headache, Medically Underserved Area, Mental Health, Pain, Population Register

Depression is one of the most common behavioral health conditions seen in primary care, with prev-

This article was externally peer reviewed.

Submitted 2 July 2008; revised 2 October 2011; accepted 10 October 2011.

From the Center for Clinical and Community Health Improvement, Austen BioInnovation Institute in Akron, Akron, OH (JEJ); Department of Community Health Sciences, Northeastern Ohio University, College of Medicine and College of Pharmacy (JEJ); Department of Statistics, The University of Akron, Akron, OH (JEJ); the Department of Family Medicine, University of Pittsburgh, Pittsburgh, PA (JES-P, CLJ).

Previous affiliation: Department of Family Medicine, University of Pittsburgh, Pittsburgh, PA (JEJ).

Funding: This study was funded by the HRSA Office of Minority Health and the Pennsylvania Department of Health, Office of Health Equity. alence data on depression indicating that within the United States almost $17 \%$ of the overall population may have a major depressive disorder at some point in their lifetime. ${ }^{1}$ The global importance of depression is reflected in the declaration by the World Health Organization that the 3 leading causes of burden of disease in 2030 are projected to include HIV/AIDS, depression, and ischemic heart disease. ${ }^{2}$ Lifetime prevalence of major depressive dis-

Prior presentation: This work was presented as a poster at the 2007 North American Primary Care Research Group Annual Meeting, Vancouver, BC.

Conflict of interest: none declared.

Corresponding author: Jeannette E. South-Paul, MD, 3518 Fifth Avenue, Pittsburgh, PA 15261 (E-mail: southpaulj@upmc.edu). 
order by race shows that the prevalence of depression in women is significantly higher than in men and that whites have a significantly earlier onset of depression than African Americans. ${ }^{3}$ Prevalence of current depression indicates that 14.4 million people $(5.3 \%)$ in the United States have depression. ${ }^{4}$ Commonly characterized symptoms of depression are sadness, fatigue, anhedonia, and sleep disturbances that last for more than 2 weeks. ${ }^{5}$

Current literature suggests a relationship between depression and physical pain. ${ }^{6,7}$ Pain and depression are mutually exacerbating, and their coexistence predicts a longer time to remission once treatment begins. ${ }^{8}$ Primary care clinicians recognize that many pain syndromes are comorbid with depression, and common physical symptoms reported with depression include headaches (14\%), ${ }^{9}$ back pain, abdominal pain (15\%), nonspecific chest pain $(11 \%)$, and generalized muscle pain. ${ }^{8,10-12}$ Family physicians and other primary care clinicians find that up to $80 \%$ of depressed patients present to their practices with these physical symptoms. McLaughlin et $\mathrm{al}^{12}$ reported findings of physical pain associated with symptoms not only of depression, but also of anxiety.

Inheritability seems to play a significant role in the prevalence of the diagnosis of depression. Depression has been known to be consistent within pedigrees, including parentage and first- and second-degree relatives. ${ }^{13}$ When considering a differential diagnosis that includes depression, a family history of depression is frequently a significant factor. ${ }^{13}$ Greden ${ }^{14}$ has reported that depression typically has an earlier symptom onset than most clinicians recognize and depression often can progress for years before being treated.

Much of what we know regarding complaints and conditions comorbid with depression results from studies of patients presenting to specialty clinics and physicians' offices. A better understanding of how depression presents in a clinically undifferentiated population that also has a representation of underrepresented minorities could improve the identification of this global condition. Recognizing common comorbid conditions and the risk factors for depression, as well as differences in presentation that may relate to the diversity of the population, would allow primary care physicians and other clinicians to predict the onset of depression more accurately and potentially intervene earlier with effective treatment and intervention. Although there is limited information in this context, particularly for minority populations, the goal of our study was to examine the relationship of depression and associated self-reported conditions in participants enrolled in a community-based research registry, a substantial number of whom were underrepresented minorities.

\section{Methods}

The study was conducted using a research registry that was created by recruiting community members who had expressed interest in participating in health education projects being conducted by the Center for Primary Care Community-Based Research and patients who were seeking or receiving care at 10 community-based primary care health centers associated with the Department of Family Medicine at the University of Pittsburgh. ${ }^{7}$ The study was reviewed and approved by the University of Pittsburgh Institutional Review Board (nos. 0408083, 0504050, 0503149, and PRO07020006). The community members were recruited from venues such as health fairs, farmers markets, and gatherings within the community.

When they expressed interest in enrollment, informed consent was obtained from each of the potential participants. This allowed their identifiable medical record information related to health status to be placed in the research registry for the purpose of facilitating retrospective research studies. $^{7,15,16}$ These retrospective research studies were directed at individual participant risk for and identification of diseases and the identification and recruitment of potential eligible subjects for participation in future research studies involving the prevention and treatment of medical conditions. Additional information regarding the methods used for recruitment and the management of the research registry have been published elsewhere. ${ }^{7,15-17}$

For the purposes of this study, responses for the following were included: current depression, anxiety, current leg pain, current severe/frequent headaches, dizziness, currently taking depression medication, currently taking anxiety medication, family history of depression, and family history of anxiety. Various planned statistical analyses were used including descriptive, univariate analyses and logistic regression. Variables statistically significant from the aforementioned univariate analyses compared 
individuals who reported depression with those with no reported depression, and they were entered into a logistic regression, using an outcome variable of current depression, for the examination of concomitant effects. Two logistic regression models (cutoff value, 0.450 ) were performed. The first logistic regression included the possible predictors of current anxiety, current leg pain, current severe/ frequent headaches, currently taking depression medication, currently taking anxiety medication, family history of depression, and family history of anxiety. The second logistic regression was performed with only current anxiety and family history of depression as independent variables. The receiver operating characteristic (ROC) analysis was used for evaluating the predicted probability of the logistic regressions. ${ }^{18-28}$ The analyses were performed using both SPSS (IBM, Chicago, IL) and SAS software (SAS Inc., Cary, NC). Statistical significance was defined as $P<.05$, but the actual significance levels are reported here where appropriate.

\section{Results}

Table 1 summarizes participants' demographics, general health, and depression-related family history and conditions. The age of participants ranged from 11 years to 91 years (mean, 46.6 years; standard deviation, 16.0; $\mathrm{N}=2421$ ). Nearly three quarters of the participants were women $(72.2 \%)$. African Americans represented $54.9 \%$ of the participants, and whites represented $38.5 \%$. The majority of participants reported on a 5-point Likert scale that their general health was either good or very good $(68.9 \%)$, with possible responses ranging from very poor, poor, fair, good, and very good.

The overall prevalence of the family histories, current conditions, and medications are shown in Figures 1 to 3 . The detailed comparison of prevalence rates of depression, anxiety, leg pains, dizziness, and headache by sex, race, and age-group are summarized in Table 2. Compared with men, women have a significantly higher prevalence of current depression $(26.6 \%$ vs. $20.2 \% ; P<.01)$, current anxiety (22.1\% vs. $14.8 \% ; P<.01)$, and current headache $(14.5 \%$ vs. $7.1 \% ; P<.01)$. Women also had a higher prevalence of a family history of depression $(38.4 \%$ vs. $24.9 \% ; P<.01)$, anxiety $(27.3 \%$ vs. $15.7 \% ; P<$ $.01)$, and of taking depression (19.9\% vs. $12.4 \% ; P<$ $.01)$ and anxiety $(12.0 \%$ vs. $8.1 \% ; P=.018)$ medications.
Table 1. Demographics, General Health, Family History, and Depression-Related Conditions $(\mathrm{N}=2421)$

\begin{tabular}{lc}
\hline Parameter & \\
\hline Age (mean years [SD]) & $46.6(16.0)$ \\
Age group (\%) & \\
$\quad<35$ years & 22.7 \\
35-50 years & 37.4 \\
51-64 years & 26.0 \\
$\geq 65$ years & 13.9 \\
Women (\%) & 72.2 \\
Race (\%) & \\
White & 38.5 \\
African American & 54.9 \\
Others & 6.6 \\
Self-reported general health (\%) & \\
Very good to good & 68.9 \\
Fair to very poor & 31.1 \\
Family history (\%) & \\
Depression & 34.1 \\
Anxiety & 23.6 \\
Current reported conditions (\%) & \\
Depression & 24.4 \\
Anxiety & 11.8 \\
Leg pain & \\
Dizziness & 17.7 \\
Headache & \\
Reported taking medicine (\%) & \\
Depression & \\
Anxiety & \\
\hline
\end{tabular}

$\mathrm{SD}$, standard deviation.

Compared with nonwhites, whites had a significantly higher prevalence of current depression (26.3\% vs. $23.8 \% ; P=.01)$, current anxiety $(25.5 \%$ vs. $16.6 \% ; P<.01)$, and current headache $(14.2 \%$ vs. $11.2 \% ; P=.015)$. Whites also had a higher prevalence of a family history of depression $(38.4 \%$ vs. $32.1 \% ; P<.01)$, anxiety $(8.9 \%$ vs. $7.7 \% ; P<$ .01 ), and of taking medications for depression $(22.4 \%$ vs. $14.8 \% ; P<.01)$ and anxiety $(15.8 \%$ vs. $7.8 \% ; P<.01)$. However, nonwhites had a higher prevalence of leg pain $(18.8 \%$ vs. $14.9 \% ; P=.042)$ and a lower prevalence of headache $(11.2 \%$ vs. $14.2 \% ; P=.015)$.

There were significant differences for most of the variables by age groups (younger than 35 years, 35-50 years, 51-64 years, 65 years and older). Compared with other age groups, the 35- to 50year-old age group had the largest prevalence of current depression $(30.5 \% ; P<.01)$, current anx- 
Figure 1. Family history of depression and anxiety.

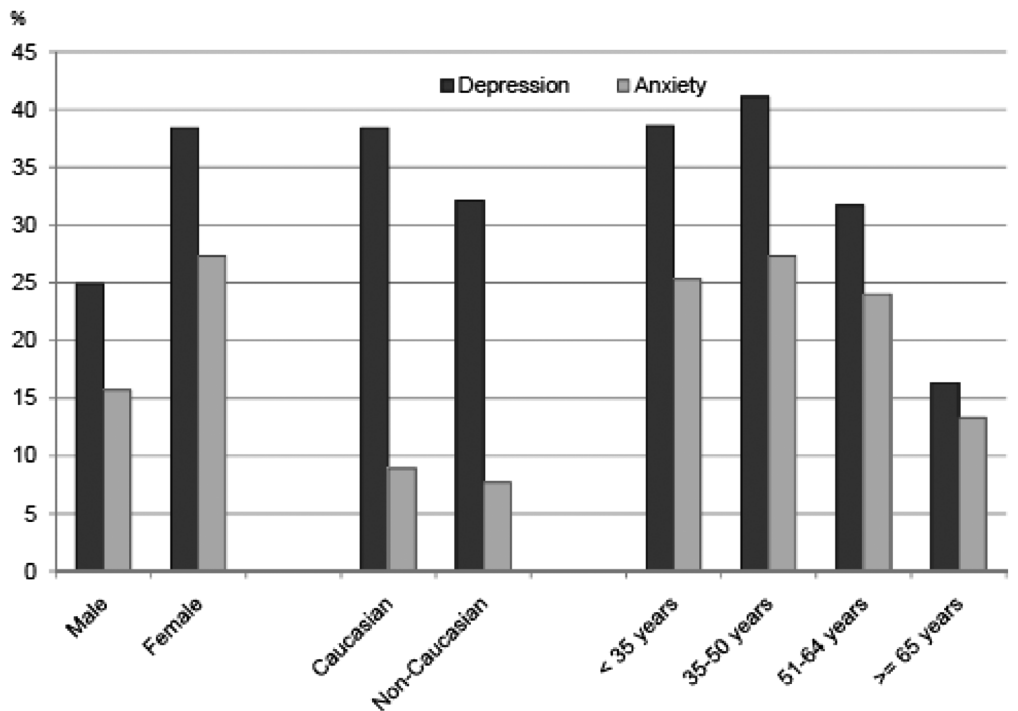

iety $(24.0 \% ; P<.01)$, and current headache $(13.6 \% ; P<.01)$. In addition, the 35 - to 50 -yearold age group had higher prevalence of taking depression medication $(22.0 \% ; P<.01)$, taking anxiety medication $(13.4 \% ; P=.019)$, and family history of depression $(41.1 \% ; P<.01)$ and anxiety (27.3\%; $P<.01)$.

The results of the first logistic regression showed that current anxiety (odds ratio, 19.825) and family history of depression (odds ratio, 5.103) were significant predictors of current depression. An ROC curve produced a 0.942 predicted probability (Figure 4) for this model. The results of the second logistic regression also showed a sig- nificant predictability value for both current anxiety (odds ratio, 12.397) and family history of depression (odds ratio, 5.504); with an ROC curve, these variables produced a 0.845 predicted probability (Figure 5).

\section{Discussion}

Physical symptoms are important presenting complaints in a diverse primary care population with specific presenting symptoms varying by sex, age, and racial group. Of the reported physical symptoms, headaches were more common among white patients and leg pain was more common among

Figure 2. Current reported conditions of depression, anxiety, leg pain, dizziness, and headache.

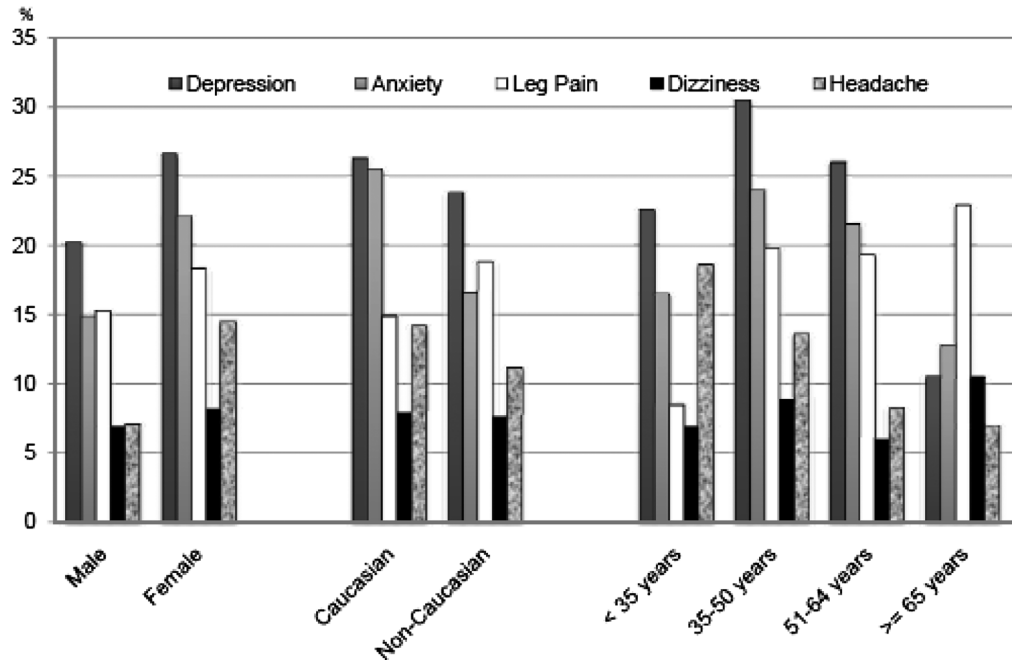


Figure 3. Reported taking medications for depression and anxiety.

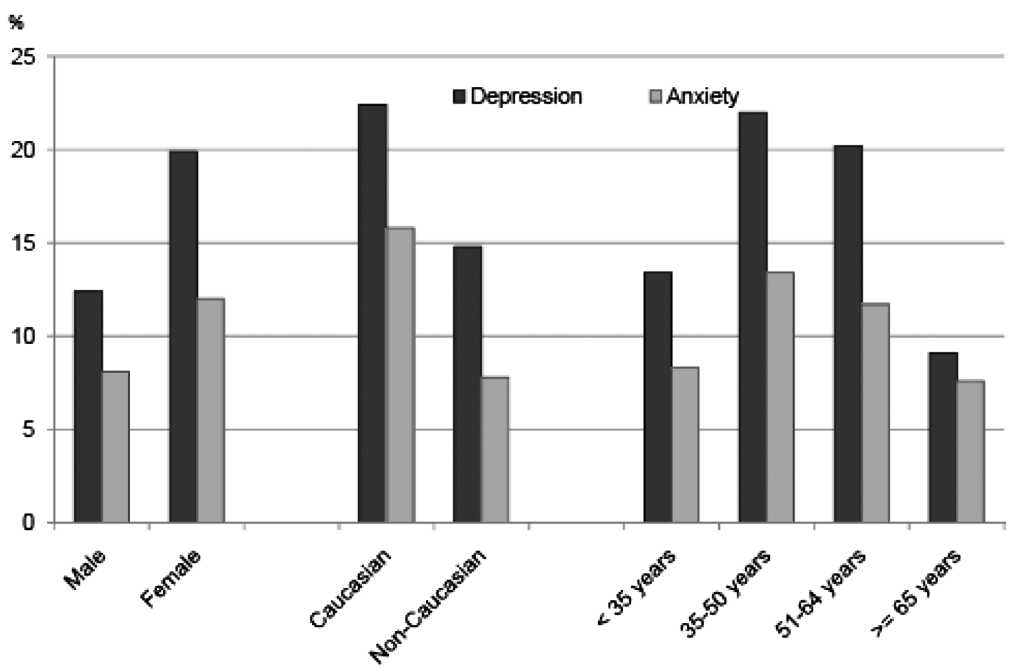

minorities. The results are consistent with previous research showing a positive relationship between the prevalence of physical pain and the incidence of depression. ${ }^{6,12,29}$ These results show that many women presenting with a variety of pain symptoms have concomitant depression. It was not the purpose of this study to determine which factors contributed to these physical symptoms, but the suggested association with depression was strong. The prevalence of depression in this diverse communitydwelling, urban population was substantially higher than what has been reported in the general population.

Many studies have focused on defined patient populations with diagnosed disease. High rates of anxiety are well recognized to occur along with depression. However, a substantial prevalence of comorbid headache was found in this population. Associations between headache and depression have been reported previously but usually are studied in patients presenting to headache clinics and those suffering from chronic daily headache more so than migraine. ${ }^{30-34}$ Chronic daily headaches are associated with psychiatric comorbidity and are seen in $3 \%$ to $5 \%$ of the population, whereas headache was seen in a much higher percentage of our population: $7.1 \%$ of men and $14.5 \%$ of women. ${ }^{32,34}$

Using data from a registry gathered from the community, our findings provide a better assessment of the underlying prevalence of depression. The results demonstrate a strong prevalence of depression and anxiety among minorities, but still less than that found in a white population $(23.8 \%$ vs. $26.3 \%$ for depression; $16.6 \%$ vs. $25.5 \%$ for anxiety). Nonetheless, the prevalence of these conditions was substantially higher in this urban, community cohort than what has been described in the general population. Furthermore, even when diagnosed with depression, minorities are less likely to take medications for depression (14.8\% vs. $22.4 \%)$ and anxiety (7.8\% vs. $15.8 \%)$.

In addition to showing a relationship to physical pain and to depression, the results of this study show a positive relationship between family history of depression, anxiety, or both and a person currently having depression. This is consistent with previous research about the impact of heredity on major depression incidence.

With the ever-growing prevalence of the disease, physicians should routinely screen patients for depression. Although sadness is the most recognized symptom of depression, it is important to note that physical pain is also a noteworthy symptom, as is physical pain for which the patient may not yet be receiving treatment. Women tend to report more pain than men, ${ }^{8}$ which is consistent with our reported findings in this cohort. In addition, pain predicts a longer time to remission and seems to be a marker for more difficult-totreat depression. ${ }^{35}$ This has been confirmed in recent research and is true when treating depression as well as when treating different pain syndromes. ${ }^{35-38}$ Depression is less likely to be treated successfully when there is untreated, comorbid pain, and pain is unlikely to be treated successfully when there is untreated, comorbid 


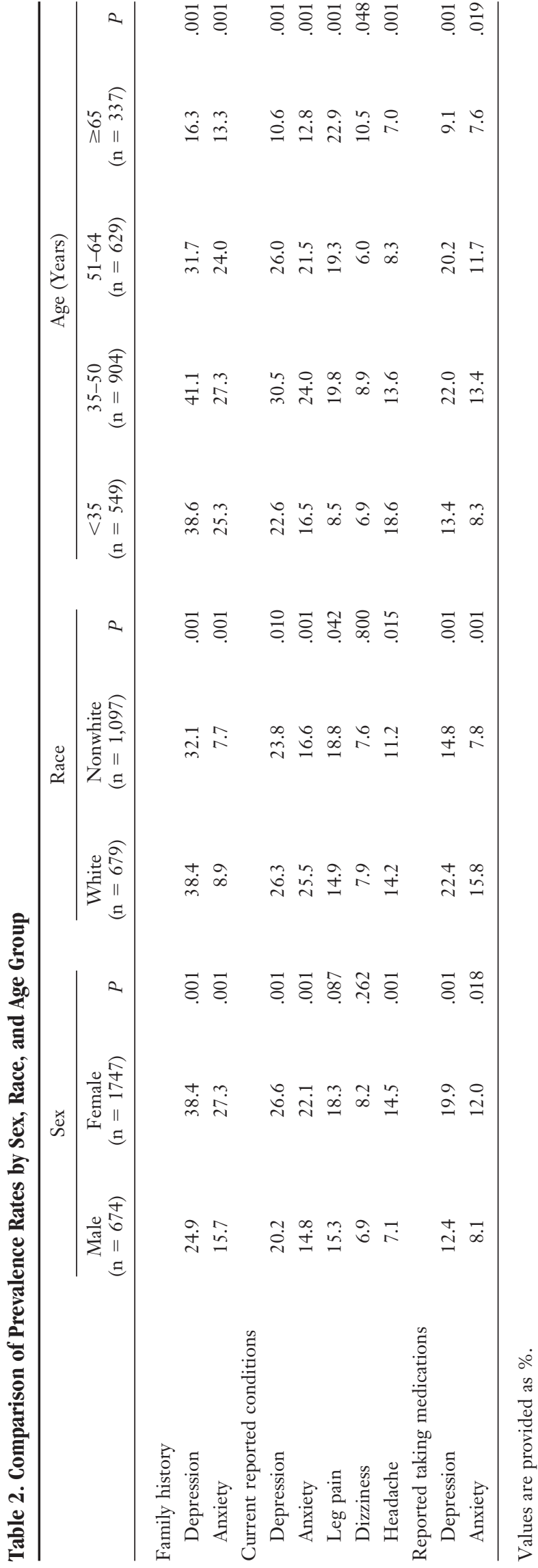

Figure 4. Predicted probability of the first logistic regression.

\section{ROC Curve}

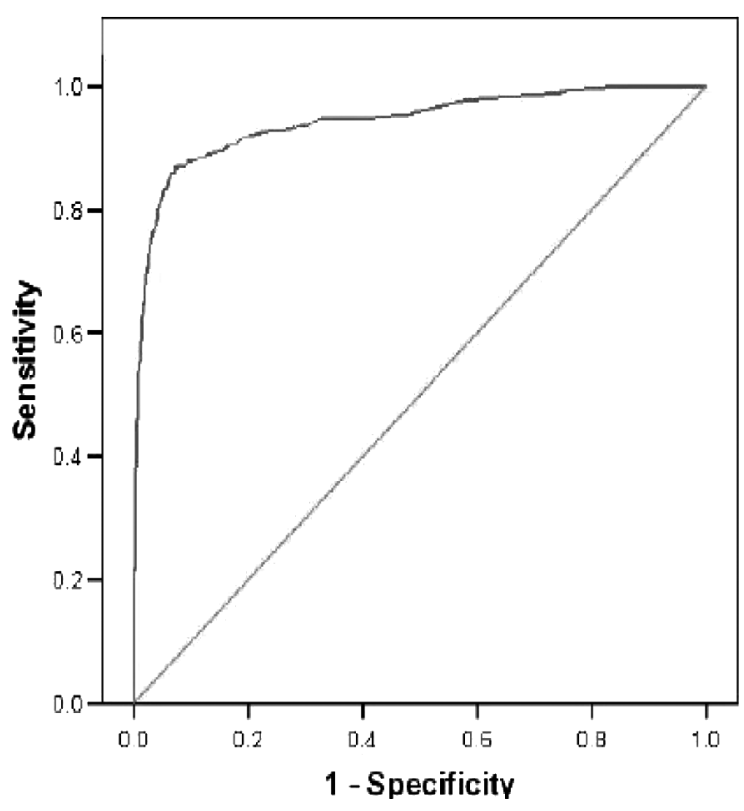

depression. Considering the diversity of this cohort and the prevalence of comorbid pain and depression, it is crucial to consider the cultural factors influencing care-seeking behavior for depression.

Some authors suggest that diverse populations (namely, younger, Latino, African American, and less educated) are more likely to report pain complaints. ${ }^{39}$ However, this finding was not evident in our cohort, which suggests that additional factors may be influencing the presenting symptoms because this is consistent with the recognition of the influence of culture in every aspect of health and health care and can serve as a focus for future studies. Perhaps the presence of what are perceived to be more serious comorbidities (chronic diseases with profound consequences when ignored) results in behavioral diagnoses and associated conditions going unaddressed. It will be valuable to determine the factors contributing to the diagnoses of depression and anxiety in culturally diverse groups. Of import would be (1) who seeks care for affective disorders, (2) which comorbidities patients associate with their behavioral diagnoses, (3) what therapies patients find acceptable (medication, psychotherapy, and complementary therapies), and (4) the influence of family history on current diagnosis and management, among others. One of the possible 
Figure 5. Predicted probability of the second logistic regression.

\section{ROC Curve}

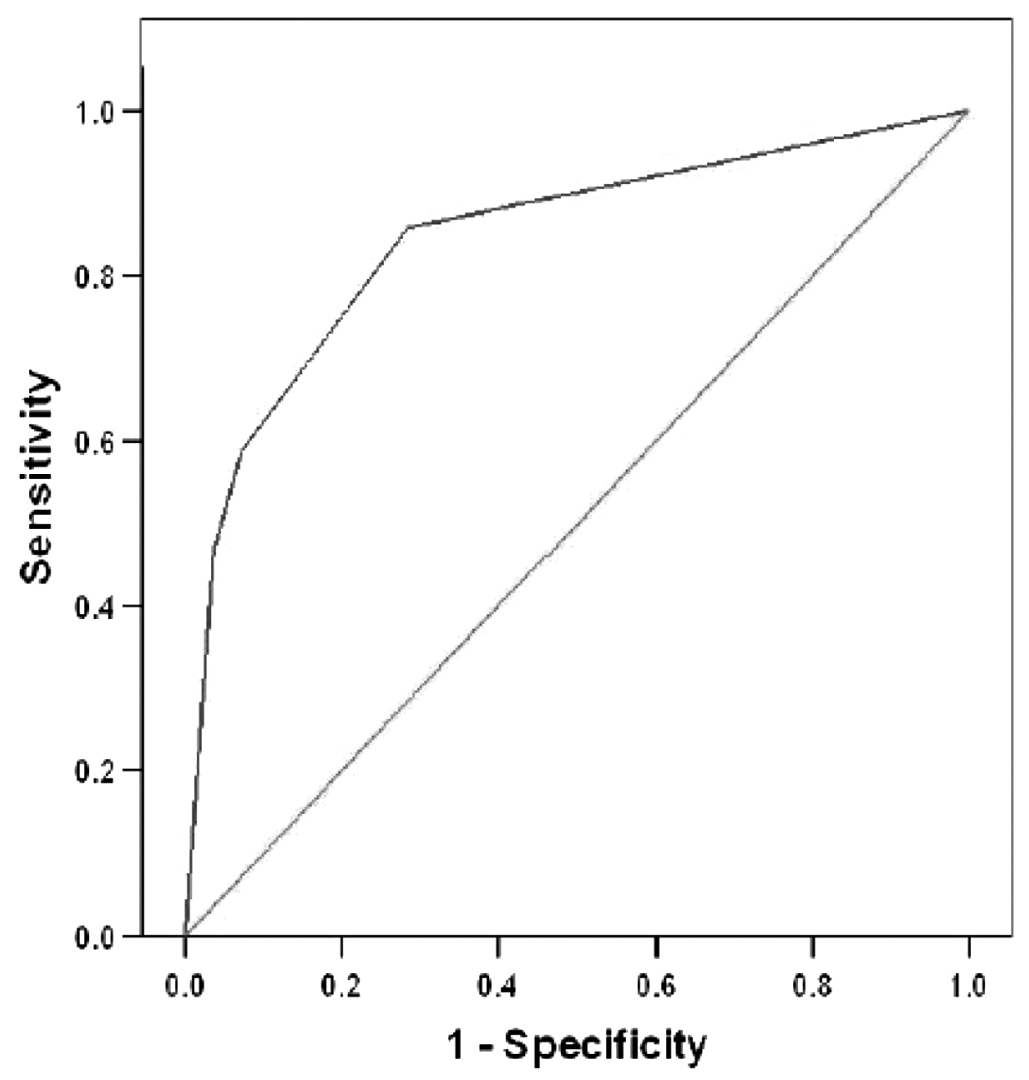

limitations of our study was that the study cohort was located in one urban area, which might not be generalizable to other locations. Nevertheless, the cohort included more than 2000 participants, of whom the majority were African Americans recruited from the community, which provided valuable information for primary care physicians.

\section{Conclusions}

Pain is commonly seen among patients with comorbid behavioral conditions. For those participating in our community-dwelling research registry and reporting pain, headache is more common in whites whereas leg pain is more common in nonwhites. Depression is less likely to be treated successfully when there is untreated, comorbid pain, and pain is unlikely to be treated successfully when there is untreated, comorbid depression. Physical pain is a noteworthy symptom of depression. Our study results suggest that family physicians should routinely screen for depression and anxiety for those who present with complaints of headache and other pain symptoms.

\section{References}

1. Kessler RC, Berglund PA, Demler O, Jin R, Merikangas KR, Walters EE. Lifetime prevalence and age-of-onset distributions of DSM-IV disorders in the National Comorbidity Survey Replication. Arch Gen Psychiatry 2005;62(6):593-602.

2. Fernandez A, Saameno JA, Pinto-Meza A, et al. Burden of chronic physical conditions and mental disorders in primary care. Br J Psychiatry 2010;196: $302-9$.

3. Riolo SA, Nguyen TA, Greden JF, King CA. Prevalence of depression by race/ethnicity: Findings from the national health and nutrition examination survey III. Am J Pub Health 2005;95(6):998-1000.

4. Better Medicine. Depression. Available from: http:// wrongdiagnosis.com/d/depression/prevalence.htm\# prevalence_intro. Accessed 20 December 2006.

5. Depression. Available from: http://www.nimh.nih. gov/health/topics/depression/index.shtml. Accessed 22 March 2012. 
6. Stahl SM. Does depression hurt? J Clin Psychiatry 2002;63:273.

7. Janosky JE, Laird SB, Qing S. Content and context of a research registry for community-based research. J Community Health 2008;33:270-8.

8. Karp JF. Pain predicts longer time to remission during treatment of recurrent depression. J Clin Psychiatry 2005;66(5):591-7.

9. Hung CI, Liu CY, Fuh JL, Juang YY, Wang SJ. Comorbid migraine is associated with a negative impact on quality of life in patients with major depression. Cephalalgia 2005;26:26-32.

10. Karp JF. Body pain and treatment response in latelife depression. Am J Geriatr Psychiatry 2005;(13)3: 188-94.

11. Nicolas G. Expression and treatment of depression among Haitian immigrant women in the United States: clinical observations. Am J Psychother 2007; 61(1):83-98.

12. McLaughlin TP, Khandker RK, Kruzikas DT, Tummala R. Overlap of anxiety and depression in a managed care population: prevalence and association with resource utilization. J Clin Psychiatry 2006; 67(8):1187-93.

13. Zimmerman M, McGlinchey JB, Young D, Chelminski I. Diagnosing major depressive disorder VII: family history as a diagnostic criterion. J Nerv Ment Dis. 2006;194(9):704-7.

14. Greden JF. Physical symptoms of depression: unmet needs. J Clin Psychiatry 2003;64(Suppl 7):5-11.

15. Janosky JE, Laird SB, Robinson JD, et al. Development of a research registry for primary care community based research. Fam Pract. 2005;1-3.

16. Janosky JE, Robinson JR. Ambulatory care quality performance measures through a primary care community-based research registry. Qual Prim Care 2007;14:243-5.

17. Janosky JE. Sun Q, Laird SB, Kostura A. The challenge of censored participants in community-based research. J Commun Engage Scholarship 2009;1(1): 73-9.

18. Zou KH, O’Malley JO, Mauri L. Receiveroperating characteristic analysis for evaluating diagnostic tests and predictive models. Circulation 2007;115:654-7.

19. Lusted LB. Signal detectability and medical decision making. Science 1971;171:1217-9.

20. Lloyd CJ. Using smooth receiver operating characteristic curves to summarize and compare diagnostic systems. J Am Stat Assoc 1998;93:1356-64.

21. Zhou XH, Obuchowski NA, McClish DK. Statistical methods in diagnostic medicine. New York, NY: Wiley \& Sons; 2002.

22. Pepe MS. The statistical evaluation of medical tests for classification and prediction. Oxford, UK: Oxford University Press; 2003.
23. Campbell G. General methodology I: advances in statistical methodology for the evaluation of diagnostic and laboratory tests. Stat Med 1994;13:499-508.

24. Shapiro DE. The interpretation of diagnostic tests. Stat Methods Med Res 1999;8:113-34.

25. Obuchowski NA. Receiver operating characteristic curves and their use in radiology. Radiology 2003; 229:3-8.

26. Eng J. Receiver operating characteristic analysis: a primer. Acad Radiol 2005;12:909-16.

27. O'Malley AJ, Zou KH, Fielding JR, Tempany CMC. Bayesian regression methodology for estimating a receiver operating characteristic curve with two radiologic applications: prostate biopsy and spiral CT of ureteral stone. Acad Radiol 2001;8:713-25.

28. Lasko TA, Bhagwat JG, Zou KH, Ohno-Machado L. The use of receiver operating characteristic curves in biomedical informatics. J Biomed Inform 2005;38: 404-15.

29. Garcia-Cebrian A, Gandhi P, Demyttenaere K, Peveler $\mathrm{R}$. The association of depression and painful physical symptoms-a review of the European literature. Eur Psychiatry 2006;21:379-88.

30. Radat F, Milowska D, Valade D. Headaches secondary to psychiatric disorders (HSPD): a retrospective study of 87 patients. Headache 2011;51:789-95.

31. Antonaci F, Nappi G, Manzoni GC, et al. Migraine and psychiatric comorbidity: a review of clinical findings. J Headache Pain 2011;12:115-25.

32. Couch JR. Update on chronic daily headache. Curr Treat Options Neurol 2011;13:41-55.

33. Okumura T, Tanno S, Ohhira M, et al. Characteristics in patients with headache in an outpatient clinic in Japan. Asia Pac Fam Med 2010;9:10.

34. Juang KD, Wang SJ, Fuh JL, et al. Comorbidity of depressive and anxiety disorders in chronic daily headache and its subtypes. Headache 2000;40: $818-23$.

35. Chou KL. Reciprocal relationship between pain and depression in older adults: evidence from the English Longitudinal Study of Aging. J Affect Disord 2007; 102(1-3):115-23.

36. Bair MJ, Robinson RL, Katon W, Kroenke K. Depression and pain comorbidity: a literature review. Arch Intern Med 2003;163(20):2433-45.

37. Deveaugh-Geiss AM, West SL, Miller WC, Sleath B, Gaynes BN, Kroenke K. The adverse effects of comorbid pain on depression outcomes in primary care patients: results from the artist trial. Pain Med 2010;11:732-41.

38. Arola HM, Nicholls E, Mallen C, Thomas E. Selfreported pain interference and symptoms of anxiety and depression in community-dwelling older adults: can a temporal relationship be determined? Eur J Pain. 2010;14:966-71.

39. Husain MM. Pain in depression: $\mathrm{STAR}^{\star} \mathrm{D}$ study findings. J Psychosom Res 2007;63(2):113-22. 